\title{
A Higgs mechanism for vector Galileons
}

\author{
Matthew Hull, ${ }^{a}$ Kazuya Koyama ${ }^{a}$ and Gianmassimo Tasinato ${ }^{a, b}$ \\ ${ }^{a}$ Institute of Cosmology \& Gravitation, University of Portsmouth, \\ Dennis Sciama Building, Portsmouth, PO1 3FX, U.K. \\ ${ }^{b}$ Department of Physics, Swansea University, \\ Swansea, SA2 8PP, U.K. \\ E-mail: matthew.hull@port.ac.uk, kazuya.koyama@port.ac.uk, \\ gianmassimo.tasinato@port.ac.uk
}

ABSTRACT: Vector theories with non-linear derivative self-interactions that break gauge symmetries have been shown to have interesting cosmological applications. In this paper we introduce a way to spontaneously break the gauge symmetry and construct these theories via a Higgs mechanism. In addition to the purely gauge field interactions, our method generates new ghost-free scalar-vector interactions between the Higgs field and the gauge boson. We show how these additional terms are found to reduce, in a suitable decoupling limit, to scalar bi-Galileon interactions between the Higgs field and Goldstone bosons. Our formalism is first developed in the context of abelian symmetry, which allows us to connect with earlier work on the extension of the Proca action. We then show how this formalism is straightforwardly generalised to generate theories with non-abelian symmetry.

KEYwords: Spontaneous Symmetry Breaking, Cosmology of Theories beyond the SM, Gauge Symmetry

ARXIV EPRINT: 1408.6871 


\section{Contents}

1 Introduction 1

2 Higgs mechanism and generalized abelian symmetry breaking 3

3 Higgs mechanism and generalized non-abelian symmetry breaking $\quad 9$

4 Conclusions 12

$\begin{array}{ll}\text { A Consistency of our Higgs higher-dimensional interactions } & 13\end{array}$

$\begin{array}{ll}\text { B Ghost free scalar-vector interactions } & 14\end{array}$

$\begin{array}{lll}\text { B.1 Bi-Galileons } & 14\end{array}$

$\begin{array}{ll}\text { B.2 Bi-vectors and the scalar-vector Lagrangian } & 16\end{array}$

\section{Introduction}

The nature of dark energy is one of the most profound open problems in Physics. Present day cosmic acceleration could be associated with some contribution to the matter energy momentum tensor, in addition to matter and radiation. Possible examples are a positive cosmological constant, or some scalar field whose dynamics make the universe accelerate as in scalar-tensor theories; see [1] for a review. Alternatively, this phenomenon can be due to an infrared modification of the gravitational interactions described by Einstein's General Relativity (GR): a recent review on this topic is given in [2]. On the other hand, Lovelock's theorem ensures that any consistent modification of the theory of GR plus cosmological constant introduces new degrees of freedom. Such theories contain additional gravitational modes, typically including scalars, that potentially mediate long-range interactions.

This implies that any theory attempting to explain dark energy (besides a pure cosmological constant) has to deal with new fields, whose interactions with matter must be suppressed at sufficiently small scales to satisfy stringent constraints from the absence of any detectible fifth force. This can be done by screening new interactions by means of either chameleon [3] or Vainshtein [4] mechanisms. Galileons are a class of scalar-tensor theories that, by exploiting the non-linearity of derivative self-interactions, are able to generate cosmological acceleration while at the same time automatically screen scalar forces at small scales via a Vainshtein mechanism [5]. Interestingly, scalars with Galileon interactions can find explicit realizations as Stückelberg fields in theories with broken local symmetries: examples are gravity theories with broken diffeomorphism invariance such as dRGT massive gravity [6], or vector theories with broken gauge symmetries such as those developed in [7, 8]. Physically, Galileon scalars are associated with the Goldstone bosons 
from broken continuous symmetries. From a theoretical point of view, the advantage of realizing Galileons is that they come with stringent consistency requirements (in particular the absence of ghosts) and this helps to reduce the size of the parameter space, which makes these set-ups more predictive than generic scalar-tensor scenarios. Moreover, the Galileonic symmetry underlying these models can play a role in protecting the structure of the theory under radiative corrections.

So far however, the symmetry in this class of theories has been broken by hand and then recovered at a second stage by adding Stückelberg fields with specific interactions. An issue with applying the Stückelberg approach to ensure gauge invariance is that it is not always easy to de-mix the physical degrees of freedom, especially in non-abelian gauge theories, and thus it can be difficult to verify whether the theory is under perturbative control within the range of interest. Moreover, unitarity problems can arise: a typical example is the scattering amplitude of W-bosons in the Standard Model, which needs to be unitarized by new physics arising below the $\mathrm{TeV}$ scale. For these reasons it would be interesting to generalize these constructions, by breaking diffeomorphisms or gauge symmetries spontaneously - for example by a Higgs mechanism - and yet still be able to recover Galileonic interactions, at least in some limits, for the available degrees of freedom in the broken phase. The advantage of spontaneous breaking is that the underlying symmetry invariance can protect and further restrict the structure of the theory and it can improve the perturbative behavior of scattering amplitudes. Moreover, it can additionally provide criteria - based on symmetry principles - to extend the abelian theory of $[7,8]$ to the non-abelian case and possibly determine couplings between the dark energy sector and standard matter, offering new avenues to test the theory. A way to spontaneously break diffeomorphism invariance to obtain dRGT massive gravity has yet to be found. However, we will show that, instead, a Higgs mechanism for vector theories with broken gauge symmetries such as those developed in $[7,8]$ can be realized. The gauge symmetry can be spontaneously broken by a Higgs scalar field acquiring a vacuum expectation value, and the theory after symmetry breaking coincides with the broken abelian gauge theory of [7]. Additionally, our Higgs mechanism can be straightforwardly extended to scenarios with non-abelian symmetry, showing that a Higgs construction can suggest new ways to straightforwardly generalize the theory of interest to interesting and quite non-trivial set-ups.

The Goldstone bosons associated with the broken symmetry are 'eaten' by the longitudinal modes of the vector (more precisely, a unitary gauge can be selected that set them to zero). However, in an appropriate decoupling limit, the dynamics of the vector longitudinal modes correspond to one of the would-be Goldstone bosons which is controlled by Galileon interactions. We show that the interactions of the scalar Higgs field itself also enjoys Galileonic symmetries, and that the Higgs-Goldstone boson system assembles into a specific bi-Galileon combination.

A Higgs mechanism, by construction, adds some new degrees of freedom to the theory, gauged under the symmetry being considered together with a non-trivial potential that spontaneously breaks this symmetry. We start in section 2 by discussing the case of abelian interactions. We consider as a fundamental degree of freedom a complex Higgs scalar field charged under the $\mathrm{U}(1)$ abelian gauge symmetry, with a classical 'Mexican hat' poten- 
tial. The new Higgs interactions that we consider correspond to higher dimensional nonrenormalizable operators, involving gauge invariant derivative self-couplings of the Higgs field. When the Higgs field sits at the minimum of its potential and acquires a vacuum expectation value $v$, the resulting theory corresponds to the vector self-interacting theory studied in [7], with parameters depending on $v$, the gauge coupling constant $g$, as well as on the parameters characterizing the higher derivative Higgs self-interactions. Moreover, when considering Higgs excitations around its minimum, one finds new scalar-vector derivative interactions - absent in the original theory that involved vector self-interactions only appearing in consistent combinations built in such a way to avoid the appearance of ghosts. This is a stringent requirement that constrains the structure of the Higgs self-interaction. We determine various examples of higher dimensional derivative self-interactions for the Higgs boson, that once expanded around the minimum of the Higgs potential lead to ghostfree derivative interactions between the vector and scalar, that generalize multi-Galileon constructions to the vector case. We show that in a suitable decoupling limit the theory reduces to a scalar bi-Galileon theory, that couples with Galileon invariant interactions the Higgs boson with the would-be Goldstone modes of the broken symmetry. In the interest of highlighting the relevance of a Vainshtein-like effect for our model, we conclude the section by briefly discussing a scenario in which the Higgs and the vector are coupled to external matter. In section 3 we straightforwardly extend our constructions to the case of non-abelian symmetry, and discuss some of its physical consequences.

As far as we are aware, this is the first example of a consistent realization of a Higgs mechanism in theories with a spontaneously broken symmetry, that lead to Galileonic theories in the remaining degrees of freedom. Our set-up can be regarded as a possible step towards finding a consistent UV completion of theories closely related to Galileons.

\section{Higgs mechanism and generalized abelian symmetry breaking}

We discuss a Higgs mechanism that spontaneously breaks an abelian symmetry, in such a way to generate a vector mass term and the class of derivative vector self-interactions studied in $[7,8]$. We work in four dimensional Minkowski space. It is well known that an abelian symmetry can be broken by a mass term controlled by a scale $m_{A}$. However, in addition to this, we can add derivative interactions for the vector field $A_{\mu}$, the simplest of which is a dimension- 4 operator weighted by a dimensionless coupling, (denoted as $\beta$ ):

$$
\mathcal{L}_{A}=-m_{A}^{2} A_{\mu} A^{\mu}-\beta A_{\mu} A^{\mu} \partial_{\rho} A^{\rho} .
$$

In addition, one can consider a handful of higher-dimensional operators with a similar structure as above. These operators break abelian gauge invariance, but are nevertheless consistent since the $A_{0}$-component of the gauge field is a constraint: its equation of motion does not contain time derivatives acting on the field. So (2.1) does not induce ghost degrees of freedom: see $[7,8]$ for more details. These systems are interesting for their cosmological applications and, as we will see, they are related to Galileons, since the dynamics of Goldstone bosons associated with the breaking of symmetry is described by Galilean interactions, at least in an appropriate decoupling limit. 
Interactions as the one in eq. (2.1) can arise by a process of spontaneous breaking of gauge symmetry via a Higgs mechanism. Let us consider a gauge invariant action for a complex scalar Higgs field with higher order derivative couplings,

$$
\begin{aligned}
\mathcal{L}_{\text {tot }}= & -\left(\mathcal{D}_{\mu} \phi\right)\left(\mathcal{D}^{\mu} \phi\right)^{*}-\frac{1}{4} F^{\mu \nu} F_{\mu \nu}-V(\phi) \\
& +\mathcal{L}_{(8)}+\mathcal{L}_{(12)}+\mathcal{L}_{(16)} .
\end{aligned}
$$

The first line contains the usual kinetic terms for scalar and vector $\left(F_{\mu \nu}=\partial_{\mu} A_{\nu}-\partial_{\nu} A_{\mu}\right)$ and the Higgs potential. The second line contains new dimension 8, 12, 16 gauge invariant operators, that are suppressed by a mass scale $\Lambda$, and describe the Higgs derivative selfinteractions associated with the pattern of spontaneous symmetry breaking that we are interested in.

The covariant derivative acting on the Higgs field contains the gauge field $A_{\mu}$, and is defined as

$$
\mathcal{D}_{\mu}=\partial_{\mu}-i g A_{\mu},
$$

with $g$ a coupling constant. The Higgs potential has the traditional 'Mexican hat' form

$$
V(\phi)=-\mu^{2} \phi \phi^{*}+\frac{\lambda}{2}\left(\phi \phi^{*}\right)^{2}
$$

and has a minimum at

$$
\langle\phi\rangle \equiv v=\left(\frac{\mu^{2}}{\lambda}\right)^{1 / 2} .
$$

We demand that Lagrangian $\mathcal{L}_{\text {tot }}$ is invariant under a $\mathrm{U}(1)$ gauge symmetry, acting on the scalar and on the vector as

$$
\begin{aligned}
\phi & \rightarrow \phi e^{i \xi}, \\
A_{\mu} & \rightarrow A_{\mu}+\frac{1}{g} \partial_{\mu} \xi,
\end{aligned}
$$

for an arbitrary function $\xi$. Under a U(1) transformation, the covariant derivative transforms as

$$
\begin{aligned}
\mathcal{D}_{\mu} \phi & \rightarrow e^{i \xi} \mathcal{D}_{\mu} \phi, \\
\mathcal{D}_{\mu} \mathcal{D}_{\mu} \phi & \rightarrow e^{i \xi} \mathcal{D}_{\mu} \mathcal{D}_{\nu} \phi .
\end{aligned}
$$

Using the transformation properties of the covariant derivative under gauge transformations, it is straightforward to check that the following tensors are gauge invariant:

$$
\begin{aligned}
L_{\mu \nu} & \equiv \frac{1}{2}\left[\left(\mathcal{D}_{\mu} \phi\right)^{*}\left(\mathcal{D}_{\nu} \phi\right)+\left(\mathcal{D}_{\nu} \phi\right)^{*}\left(\mathcal{D}_{\mu} \phi\right)\right], \\
P_{\mu \nu} & \equiv \frac{1}{2}\left[\phi^{*} \mathcal{D}_{\mu} \mathcal{D}_{\nu} \phi+\phi\left(\mathcal{D}_{\mu} \mathcal{D}_{\nu} \phi\right)^{*}\right], \\
Q_{\mu \nu} & \equiv \frac{i}{2}\left[\phi\left(\mathcal{D}_{\mu} \mathcal{D}_{\nu} \phi\right)^{*}-\phi^{*} \mathcal{D}_{\mu} \mathcal{D}_{\nu} \phi\right] .
\end{aligned}
$$

Notice that $P_{\mu \nu}$ and $Q_{\mu \nu}$ are formed by second covariant derivatives: these contain derivatives of the vectors, that are needed to build derivative vector self-interactions as in eq. (2.1). 
Together with the totally antisymmetric $\varepsilon$-tensor in four dimensions (with $\varepsilon_{0123}=1$ ), the previous tensors are the ingredients we use to define the operators $\mathcal{L}_{(8),(12),(16)}$ introduced in the second line of eq. (2.2) as

$$
\begin{aligned}
\mathcal{L}_{(8)} & =\frac{1}{2 ! \Lambda^{4}} \varepsilon^{\alpha \beta \mu_{1} \mu_{2}} \varepsilon_{\alpha \beta \nu_{1} \nu_{2}}\left[\alpha_{(8)} L_{\mu_{1}}^{\nu_{1}} P_{\mu_{2}}^{\nu_{2}}+\beta_{(8)} L_{\mu_{1}}^{\nu_{1}} Q_{\mu_{2}}^{\nu_{2}}\right] \\
\mathcal{L}_{(12)} & =\frac{1}{\Lambda^{8}} \varepsilon^{\alpha \mu_{1} \mu_{2} \mu_{3}} \varepsilon_{\alpha \nu_{1} \nu_{2} \nu_{3}}\left[\alpha_{(12)} L_{\mu_{1}}^{\nu_{1}} P_{\mu_{2}}^{\nu_{2}} P_{\mu_{3}}^{\nu_{3}}+\beta_{(12)} L_{\mu_{1}}^{\nu_{1}} Q_{\mu_{2}}^{\nu_{2}} Q_{\mu_{3}}^{\nu_{3}}\right] \\
\mathcal{L}_{(16)} & =\frac{1}{\Lambda^{12}} \varepsilon^{\mu_{1} \mu_{2} \mu_{3} \mu_{4}} \varepsilon_{\nu_{1} \nu_{2} \nu_{3} \nu_{4}}\left[\alpha_{(16)} L_{\mu_{1}}^{\nu_{1}} P_{\mu_{2}}^{\nu_{2}} P_{\mu_{3}}^{\nu_{3}} P_{\mu_{4}}^{\nu_{4}}+\beta_{(16)} L_{\mu_{1}}^{\nu_{1}} Q_{\mu_{2}}^{\nu_{2}} Q_{\mu_{3}}^{\nu_{3}} Q_{\mu_{4}}^{\nu_{4}}\right]
\end{aligned}
$$

that are weighted by dimensionless parameters $\alpha_{(i)}, \beta_{(i)}$, and suppressed by an energy scale $\Lambda$ to the appropriate powers. We present in appendix A arguments that show that these operators lead to equations of motion with at most two space-time derivatives, analogously to what happens for standard Galileons [5]. Indeed, the $\varepsilon$-tensors present in the above definitions have been introduced to automatically avoid the emergence of ghost degrees of freedom. Similar gauge invariant Higgs Lagrangians were also studied in [9, 10]. Notice that all these operators are higher-dimensional and hence apparently non-renormalizable: we will return to this point at the very end of this section.

To understand the physical consequences of these new self-interactions, it is convenient to decompose the complex scalar into its norm and phase:

$$
\phi=\varphi e^{i g \pi},
$$

where $\varphi, \pi$ are two real fields. $\varphi$ does not transform under $\mathrm{U}(1)$ gauge symmetry, while the field $\pi$ transforms non-linearly as $\pi \rightarrow \pi+\frac{\xi}{g}$ : the phase $\pi$ behaves as the would-be Goldstone boson for the broken abelian symmetry. Hence defining the gauge invariant combination

$$
\hat{A}_{\mu} \equiv A_{\mu}-\partial_{\mu} \pi
$$

we can express the covariant derivatives as

$$
\begin{aligned}
\mathcal{D}_{\mu} \phi & =\left[\partial_{\mu} \varphi-i g \varphi \hat{A}_{\mu}\right] e^{i g \pi} \\
\mathcal{D}_{\mu} \mathcal{D}_{\nu} \phi & =\left[\partial_{\mu} \partial_{\nu} \varphi-i g \varphi \partial_{\mu} \hat{A}_{\nu}-i g \hat{A}_{\mu} \partial_{\nu} \varphi-i g \hat{A}_{\nu} \partial_{\mu} \varphi-g^{2} \varphi \hat{A}_{\mu} \hat{A}_{\nu}\right] e^{i g \pi},
\end{aligned}
$$

with the pieces inside the square parenthesis invariant under the gauge transformations. It is important to stress that using this Higgs construction the would-be Goldstone fields combine automatically with the vectors and appear in the action only in the gauge invariant combination (2.17).

Using these relations, the operators defined in eqs. (2.13)-(2.15) can be expressed as

$$
\begin{aligned}
L_{\mu \nu} & =\partial_{\mu} \varphi \partial_{\nu} \varphi+g^{2} \varphi^{2} \hat{A}_{\mu} \hat{A}_{\nu} \\
P_{\mu \nu} & =\varphi \partial_{\mu} \partial_{\nu} \varphi-g^{2} \varphi^{2} \hat{A}_{\mu} \hat{A}_{\nu} \\
Q_{\mu \nu} & =\frac{g}{2}\left[\partial_{\mu}\left(\varphi^{2} \hat{A}_{\nu}\right)+\partial_{\nu}\left(\varphi^{2} \hat{A}_{\mu}\right)\right]
\end{aligned}
$$


which shows that they are symmetric in their two indexes. It is straightforward to plug these expressions into eqs. (2.13) to derive explicit forms for the Lagrangians $\mathcal{L}_{(8),(12),(16)}$, by also using the following identity involving contractions of the $\varepsilon$-tensors:

$$
\varepsilon_{\alpha_{1} \ldots \alpha_{4-n} \alpha_{1} \ldots \alpha_{n}} \varepsilon^{\alpha_{1} \ldots \alpha_{4-n} \beta_{1} \ldots \beta_{n}}=-(4-n) ! n ! \delta_{\alpha_{1}}^{\left[\beta_{1}\right.} \ldots \delta_{\alpha_{n}}^{\left.\beta_{n}\right]} .
$$

where $[\ldots]$ denotes weighted index anti-symmetrization. For example, let us focus on the lower dimensional interaction contained in $\mathcal{L}_{(8)}$, proportional to the dimensionless coefficient $\beta_{(8)}$. We get

$$
\begin{aligned}
\mathcal{L}_{(8)} & =-\frac{\beta_{(8)}}{\Lambda^{4}}\left(L_{\rho}^{\rho} Q_{\sigma}^{\sigma}-L_{\mu}^{\nu} Q_{\nu}^{\mu}\right), \\
& =-\frac{g \beta(8)}{\Lambda^{4}}\left(\partial_{\mu} \varphi \partial^{\nu} \varphi+g^{2} \varphi^{2} \hat{A}_{\mu} \hat{A}^{\nu}\right) \partial_{\rho}\left(\varphi^{2} \hat{A}^{\sigma}\right)\left(\delta_{\nu}^{\mu} \delta_{\sigma}^{\rho}-\delta_{\nu}{ }^{\rho} \delta_{\sigma}^{\mu}\right) .
\end{aligned}
$$

This expression is manifestly gauge invariant, and describes the interactions between the norm $\varphi$ of the Higgs field and the gauge-invariant combination of vector and wouldbe Goldstone bosons. Additional dimension- 8 operators proportional to $\alpha_{(8)}$ could be included, that lead to other interactions between gauge fields and first derivatives of the scalar $\varphi$; these are of less interest in the present context, so we ignore them here. Analogous expressions can be straightforwardly obtained for $\mathcal{L}_{(12)}, \mathcal{L}_{(16)}$ : the resulting formulae are however cumbersome so we will not write them explicitly. We instead move on to discuss some phenomenological aspects of the Higgs interactions associated with $\mathcal{L}_{(8)}$.

As we explained, our main motivation is to generate, by the phenomenon of spontaneous symmetry breaking, the vector self-interactions of eq. (2.1) and their generalizations discussed in $[7,8]$. The phenomenon of spontaneous symmetry breaking is associated with the Higgs developing a vacuum expectation value $v$ as in eq. (2.5), and acquiring non-trivial dynamics when fluctuating around the minimum of its potential. In order to study the dynamics of Higgs fluctuations, it is convenient to expand the norm of the Higgs around the minimum $v$ of the potential, and write

$$
\varphi=\left(v+\frac{h}{\sqrt{2}}\right)
$$

which allows us to canonically normalize the Higgs fluctuations $h$. By applying this expansion, the initial Lagrangian $\mathcal{L}_{\text {tot }}$ - including only the $\beta_{(8)}$ contribution to $\mathcal{L}_{(8)}$ written in eq. $(2.25)$ - results

$$
\begin{aligned}
\mathcal{L}_{\text {tot }}= & -\frac{1}{4} F_{\mu \nu} F^{\mu \nu}-m_{A}^{2} \hat{A}^{2}-\tilde{\beta} \hat{A}_{\mu} \hat{A}^{\mu} \partial_{\rho} \hat{A}^{\rho} \\
& -\frac{1}{2}(\partial h)^{2}-\frac{1}{2} m_{h}^{2} h^{2}-\frac{\sqrt{\lambda} m_{h}}{2} h^{3}-\frac{\lambda}{8} h^{4}-\sqrt{2} g m_{A} h \hat{A}_{\mu} \hat{A}^{\mu}-\frac{g^{2}}{2} h^{2} \hat{A}_{\mu} \hat{A}^{\mu} \\
& +\frac{4 g \tilde{\beta}}{3 m_{A}}\left(\sqrt{2} h+\frac{3 g}{2 m_{A}} h^{2}+\frac{g^{2}}{\sqrt{2} m_{A}^{2}} h^{3}+\frac{g^{3}}{8 m_{A}^{3}} h^{4}\right)\left(\hat{A}_{\mu} \hat{A}^{\nu} \partial_{\nu} \hat{A}^{\mu}-\hat{A}_{\mu} \hat{A}^{\mu} \partial_{\rho} \hat{A}^{\rho}\right) \\
& +\frac{\tilde{\beta}}{3 m_{A}^{2}}\left(1+\frac{\sqrt{2} g}{m_{A}} h+\frac{g^{2}}{2 m_{A}^{2}} h^{2}\right)\left(\partial_{\mu} h \partial^{\nu} h \partial_{\nu} \hat{A}^{\mu}-\partial_{\mu} h \partial^{\mu} h \partial_{\rho} \hat{A}^{\rho}\right),
\end{aligned}
$$


with

$$
\begin{aligned}
m_{A} & =g v, \\
\tilde{\beta} & =\frac{3 g^{3} \beta_{(8)} v^{4}}{2 \Lambda^{4}}, \\
m_{h} & =\sqrt{2 \lambda} v,
\end{aligned}
$$

where we have neglected the field-independent part of the potential, that contributes to the cosmological constant.

The previous Lagrangian is fully gauge invariant, being expressed in terms of the gauge invariant combination given in eq. (2.17), and describes the dynamics of four degrees of freedom, two scalars and a massless vector. Choosing the unitary gauge $\pi=0$ enables us to analyze the dynamics of the physical degrees of freedom: the Higgs scalar $h$ and a massive gauge boson $A_{\mu}$ (again, with a total of four degrees of freedom). Working in the physically transparent unitary gauge, one finds that the previous Lagrangian eq. (2.27) leads to several interesting interactions.

In the first two lines one finds renormalizable interactions described by (up to) dimension- 4 operators: the the Higg's vev, $v$, gives a mass to the gauge field, $m_{A}=g v$, and provides the simplest example of a derivative vector self-interaction: that of eq. (2.1), which was studied in $[7,8]$. Hence, the phenomenon of spontaneous symmetry breaking automatically generates the desired vector derivative self-interactions; the dimensionless coupling constant $\tilde{\beta}$ in front of this derivative operator depends on the ratio of the Higgs vev $v$ and the scale $\Lambda$, see eq. (2.29).

On the other hand, we discover that in addition to these renormalizable derivative vector self-interactions, this Lagrangian contains new higher dimensional operators between the physical Higgs field $h$ and the gauge field, contained in the last two lines of eq. (2.27). The couplings that govern those interactions are fixed by the mechanism of symmetry breaking and gauge invariance, and are suppressed by a mass scale corresponding to the vector mass $m_{A}$ to appropriate powers. Notice that all these new higher dimensional interactions are derived from our initial Lagrangian, and consequently are ghost-free since the associated equations of motion contain at most two space-time derivatives. It is indeed straightforward to show that for all these interactions the $A_{0}$ component of the gauge field remains a constraint, and the equations of motion for all the fields contain at most two space-time derivatives (including the new vector-scalar interactions in the last line of (2.27)). One can further generalize these results by including the Lagrangians $\mathcal{L}_{12}$ and $\mathcal{L}_{16}$, that lead to the complete set of derivative vector interactions discussed in [7], and in addition to new scalar-vector interactions that generalize the last line of eq. (2.27), see appendix B for details on how they are constructed.

It would be very interesting to study the observational effects of all these new operators: since they are suppressed by powers of $m_{A}$, they can lead to sizeable effects if $m_{A}$ is not large. However, screening mechanisms might occur, similar to what happens with the Vainshtein effect and Galileon interactions in gravitational set-ups. The complete phenomenology of the previous system along these lines goes beyond the scope of this work, 
but let us develop some intriguing relations between the previous system and Galileons. We return to the fully gauge invariant Lagrangian (2.27) before choosing any gauge, with the aim to study the dynamics of would-be Goldstone bosons. In [7] it was shown that a decoupling limit exists in which the dynamics of the Goldstone bosons $\pi$ is described by Galileonic derivative self-interactions. This is a regime where some kind of equivalence theorem should hold, with the physics of the Goldstone bosons being equivalent to that of the longitudinal polarization of the vectors (see for example [11]). In our Higgs set-up, we can do one step further: we show that in this decoupling limit, not only do the Goldstone self-interactions preserve Galileon invariance by themselves, but in addition they acquire new derivative couplings with the Higgs field $h$. These automatically preserve the Galileon symmetry by assembling into bi-Galileon combinations.

To exhibit these features, the limit we have to consider is

$$
g \rightarrow 0, \quad \lambda \rightarrow 0, \quad \beta_{(8)} \rightarrow 0, \quad v \rightarrow \infty,
$$

such that

$$
m_{A} \rightarrow 0, \quad m_{h} \rightarrow 0, \quad \tilde{\beta} \rightarrow 0, \quad \frac{\tilde{\beta}}{m_{A}^{3}}=\text { fixed } \equiv \frac{1}{\Lambda_{g}^{3}},
$$

where $\Lambda_{g}$ is a mass scale that, as we will see in a moment, is associated with the strength of the Galileon interactions. Notice that the previous limits imply that $g / m_{A}=1 / v \rightarrow 0$. In order to have a correctly normalized kinetic term for the Goldstone boson $\pi$ we have to rescale this field, and define $\pi=\hat{\pi} /\left(\sqrt{2} m_{A}\right)$. Indeed the second term in the first line of (2.27) becomes, in the limit (2.31),

$$
\begin{aligned}
-m_{A}^{2}\left(A_{\mu}-\partial_{\mu} \pi\right)^{2} & =-\left(m_{A} A_{\mu}-\frac{1}{\sqrt{2}} \partial_{\mu} \hat{\pi}\right)^{2} \\
& \rightarrow-\frac{1}{2}\left(\partial_{\mu} \hat{\pi}\right)^{2}
\end{aligned}
$$

so the Goldstone boson acquires a standard kinetic term. In the limits $(2.31),(2.32)$, when expressed in terms of the canonically normalized Goldstone field $\hat{\pi}$, the total Lagrangian $\mathcal{L}_{\text {tot }}$ reduces to

$$
\begin{aligned}
\mathcal{L}_{\text {tot }}= & -\frac{1}{4} F_{\mu \nu} F^{\mu \nu}-\frac{1}{2}\left(\partial_{\mu} h\right)^{2}-\frac{1}{2}\left(\partial_{\mu} \hat{\pi}\right)^{2}-\frac{1}{\Lambda_{g}^{3}}\left(\partial_{\mu} \hat{\pi} \partial^{\mu} \hat{\pi}\right) \square \hat{\pi} \\
& -\frac{1}{3 \Lambda_{g}^{3}}\left(\partial_{\mu} h \partial^{\mu} h \square \hat{\pi}-\partial_{\mu} h \partial^{\nu} h \partial_{\nu} \partial^{\mu} \hat{\pi}\right) .
\end{aligned}
$$

Hence, as announced, in this decoupling limit the Lagrangian acquires a bi-Galileon structure, and the physical Higgs itself acquires bi-Galileon couplings ${ }^{1}[12,13]$ with the Goldstone boson describing the dynamics of the longitudinal vector polarization. The connection that we pointed out with Galileons can help to render the structure of the theory stable under radiative corrections. Galileon Lagrangians are known to enjoy powerful nonrenormalization theorems $[14,15]$ that might be applied in the present context to protect

\footnotetext{
${ }^{1}$ The above bi-Galileon interaction corresponds to (B.16) in appendix B but with $h$ and $\pi$ exchanged.
} 
the size of the higher dimensional operators $\mathcal{L}_{(8),(12),(16)}$ that we introduced in this section. We leave for future work the analysis of this point and move on to briefly discuss the possible phenomenological consequences and relevance of such interactions.

We can think to two different ways in which the Higgs field can couple to matter, that would allow to exploit the bi-Galileon interactions. The first is a direct coupling of the Higgs $\phi$ to the trace of the energy momentum tensor $T$ via operators that respect gauge invariance such as for example $\phi^{*} \phi T$. In the case in which the Higgs scalar of our model is very light - as might be required for cosmological applications - such couplings could be associated with a long range force that needs to be screened. In our set-up we have shown that, in an appropriate regime, the Higgs scalar combines with the longitudinal polarization of the vector to form bi-Galileon derivative combinations. These non-linear operators can then lead to a Vainshtein mechanism that is able to suppress the aforementioned long range force.

Other possible couplings involve derivative operators. An example among others is a gauge invariant coupling of the form $\left(\mathcal{D}_{\mu} \phi\right)^{*}\left(\mathcal{D}^{\mu} \phi\right) T$, where the $\mathcal{D}_{\mu}$ is a covariant derivative containing gauge fields (see eq (2.18)). Once the covariant derivatives are expanded, such a combination leads among others to operators of the form $A_{\mu} A^{\mu} T$, that couple vectors to the energy momentum tensor. More generally, one could generalize the derivative disformal couplings of scalars to matter proposed by Bekenstein [16], by promoting the standard derivative to covariant derivatives. It would be interesting to explore in detail the phenomenology of these derivative couplings. We can imagine that they could lead to long range forces, since the Higgs and the vector longitudinal polarization are derivatively coupled to the energy momentum tensor. The bi-Galileon self-interactions discussed above can then provide the Vainshtein mechanism needed to screen them.

These arguments of course only scratch the surface of the possible couplings of our Higgs field to matter and their phenomenological consequences. We hope to return to this subject with a separate detailed publication.

\section{Higgs mechanism and generalized non-abelian symmetry breaking}

The Higgs construction that we developed in the abelian case can be directly extended to the non-abelian case. This is interesting because, applying the Stückelberg approach in this case would be more laborious than in the abelian set-up. Again we focus on theories that contain dimension-8 operators with derivative self-interactions of the Higgs field. We investigate theories that spontaneously break non-abelian symmetries, leading to consistent derivative self-interactions for gauge vectors, and generalizing the abelian symmetry breaking case discussed in the previous section and in [7]. Instead of providing a fully general treatment, we concentrate on a representative example to make clear our arguments.

We consider an $\mathrm{SU}(2)$ theory with a doublet of complex scalars $\phi=\left\{\phi^{\alpha}\right\}$, with $\alpha=1,2$ transforming in the fundamental representation. The construction of a Higgs model for this theory, which spontaneously breaks the $\mathrm{SU}(2)$ symmetry, is a standard textbook example, see e.g. [17]. Here we consider additional derivative self-interactions of the Higgs field, that lead to derivative self-interactions of the gauge vectors. 
The Lagrangian we are interested in, is invariant under the non-abelian SU(2) symmetry, and is written,

$$
\mathcal{L}_{\mathrm{SU}(2)}=-\left(\mathcal{D}^{\mu} \phi\right)^{\dagger} \mathcal{D}_{\mu} \phi-V(\phi)-\frac{1}{2} \operatorname{tr}\left[F_{\mu \nu} F^{\mu \nu}\right]+\mathcal{L}_{(8)}^{\mathrm{SU}(2)}
$$

The field $\phi$ is our Higgs, that as stated above is a doublet under the SU(2) symmetry; the covariant derivative acts on its components as

$$
\left(\mathcal{D}_{\mu} \phi\right)^{\alpha}=\partial_{\mu} \phi^{\alpha}-i g A_{\mu}^{a}\left(T^{a}\right)_{\beta}^{\alpha} \phi^{\beta},
$$

where $T^{a}$ are the generators in the fundamental representation, that for $\mathrm{SU}(2)$ are proportional to the Pauli matrices, $T^{a}=\sigma^{a} / 2$. The non-abelian transformation acts as

$$
\begin{aligned}
\phi & \rightarrow U \phi, \\
A_{\mu} & \rightarrow U A_{\mu} U^{\dagger}-\frac{i}{g}\left(\partial_{\mu} U\right) U^{\dagger},
\end{aligned}
$$

with $A_{\mu} \equiv A_{\mu}^{a} T^{a}$, and the transformation group element is $U \equiv \exp \left[i g \theta^{a}(x) T^{a}\right]$. The covariant derivative (3.2) transforms as expected

$$
\left(\mathcal{D}_{\mu} \phi\right) \rightarrow U\left(\mathcal{D}_{\mu} \phi\right)
$$

The field strength associated with the vector potential is defined as

$$
F_{\mu \nu}=\partial_{\mu} A_{\nu}-\partial_{\nu} A_{\mu}-i g\left[A_{\mu}, A_{\nu}\right]
$$

and transforms as

$$
F_{\mu \nu} \rightarrow U F_{\mu \nu} U^{\dagger}
$$

the corresponding gauge invariant vector kinetic term is

$$
-\frac{1}{2} \operatorname{tr}\left[F_{\mu \nu} F^{\mu \nu}\right]=-\frac{1}{4} F_{\mu \nu}^{a} F^{a \mu \nu}
$$

where we used the following identity valid for fundamental representations of the gauge group $\operatorname{tr}\left[T^{a} T^{b}\right]=\frac{1}{2} \delta^{a b}$. The Higgs potential is written as

$$
V(\phi)=\lambda\left(\phi \phi^{\dagger}-v^{2}\right)^{2}
$$

and is invariant under the unitary transformations that we are considering. It is characterized by a family of degenerate vacua, with $\phi \phi^{\dagger}=v^{2}$, that spontaneously break the gauge symmetry.

The dimension-8 Lagrangian $\mathcal{L}_{(8)}^{\mathrm{SU}(2)}$ in the second line of (3.1), responsible for breaking the non-abelian symmetry in such a way to generate consistent derivative vector selfinteractions, is constructed similarly to what was done for the case of abelian symmetry in the previous section. We define the gauge invariant tensor combinations

$$
L_{\mu \nu} \equiv \frac{1}{2}\left[\left(\mathcal{D}_{\mu} \phi\right)^{\dagger}\left(\mathcal{D}_{\nu} \phi\right)+\left(\mathcal{D}_{\nu} \phi\right)^{\dagger}\left(\mathcal{D}_{\mu} \phi\right)\right]
$$




$$
Q_{\mu \nu} \equiv \frac{i}{2}\left[\phi\left(\mathcal{D}_{\mu} \mathcal{D}_{\nu} \phi\right)^{\dagger}-\phi^{\dagger} \mathcal{D}_{\mu} \mathcal{D}_{\nu} \phi\right]
$$

built in terms of the Higgs doublet $\phi$. Then,

$$
\mathcal{L}_{(8)}^{\mathrm{SU}(2)} \equiv-\frac{\beta}{\Lambda^{4}}\left[L_{\rho}^{\rho} Q_{\sigma}^{\sigma}-L_{\mu}^{\nu} Q_{\nu}^{\mu}\right]
$$

with $\beta$ a dimensionless coupling constant, and $\Lambda$ a scale. For the very same arguments discussed in the abelian case, this dimension- 8 operator is gauge invariant, and consistent since it does not introduce ghost degrees of freedom.

To proceed, we recall that $\mathrm{SU}(2)$ transformations are characterized by three free parameters, while our Higgs field has four independent real components. At this stage, we can use the gauge freedom to fix a unitary gauge and eliminate three of the Higgs four components. We write

$$
\phi=\left(\begin{array}{c}
0 \\
v+\frac{1}{\sqrt{2}} h
\end{array}\right)
$$

with $\pi$ a real scalar field. The covariant derivative acting on the Higgs becomes

$$
D_{\mu} \phi=\frac{1}{\sqrt{2}}\left(\begin{array}{c}
0 \\
\partial_{\mu} h
\end{array}\right)-i \frac{g}{2}\left(v+\frac{1}{\sqrt{2}} h\right)\left(\begin{array}{c}
A_{\mu}^{1}-i A_{\mu}^{2} \\
-A_{\mu}^{3}
\end{array}\right) .
$$

On the other hand, the second covariant derivative on the complex scalar $\phi$ acts as

$$
\begin{aligned}
D_{\nu} D_{\mu} \phi= & \partial_{\nu} \partial_{\mu} \phi^{\alpha}-i g\left(\partial_{\nu} A_{\mu}^{c}\right)\left(T^{c}\right)_{\gamma}^{\alpha} \phi^{\gamma}-i g A_{\mu}^{c}\left(T^{c}\right)_{\gamma}^{\alpha} \partial_{\nu} \phi^{\gamma}-i g A_{\nu}^{c}\left(T^{c}\right)_{\gamma}^{\alpha} \partial_{\mu} \phi^{\gamma} \\
& -g^{2} A_{\nu}^{a} A_{\mu}^{b}\left(T^{a}\right)_{\beta}^{\alpha}\left(T^{b}\right)_{\gamma}^{\beta} \phi^{\gamma} .
\end{aligned}
$$

Plugging these ingredients in the expression (3.1) for $\mathcal{L}_{\mathrm{SU}(2)}$ and expanding, we find the following Lagrangian for the Higgs field $h$, the vectors $A_{\mu}^{a}$, and their couplings (sum over repeated indexes)

$$
\begin{aligned}
\mathcal{L}_{\mathrm{SU}(2)}= & -\frac{1}{4} F_{\mu \nu}^{a} F^{a \mu \nu}-\frac{g^{2} v^{2}}{4}\left(A_{\mu}^{a} A^{a \mu}\right)-\frac{\beta g^{3} v^{4}}{8 \Lambda^{4}}\left[\left(A_{\mu}^{a} A^{a \mu}\right) \partial_{\nu} A^{3 \nu}-\left(A_{\mu}^{a} A_{\nu}^{a}\right) \partial^{\mu} A^{3 \nu}\right] \\
- & \frac{1}{2} \partial_{\mu} h \partial^{\mu} h-2 \lambda v^{2} h^{2}-\sqrt{2} \lambda v h^{3}-\frac{\lambda}{4} h^{4} \\
- & -\frac{\beta g v^{2}}{4 \Lambda^{4}}\left(\partial_{\mu} h \partial^{\mu} h \partial_{\nu} A^{3 \nu}-\partial_{\mu} h \partial^{\nu} h \partial_{\nu} A^{3 \mu}\right)\left(1+\frac{\sqrt{2} h}{v}+\frac{h^{2}}{2 v^{2}}\right) \\
- & \frac{\beta g^{3} v^{3}}{4 \sqrt{2} \Lambda^{4}}\left(h+\frac{3 h^{2}}{2 \sqrt{2} v}+\frac{h^{3}}{2 v^{2}}+\frac{h^{4}}{8 \sqrt{2} v^{3}}\right) \times \\
& \times\left[\left(A_{\mu}^{a} A^{a \mu}\right) \partial_{\nu} A^{3 \nu}+A^{a \mu} A^{3 \nu} \partial_{\mu} A_{\nu}^{a}+A_{\mu}^{a} A^{3 \mu} \partial_{\nu} A^{a \nu}\right. \\
& \left.\quad-A^{a \mu} A^{a \nu} \partial_{\mu} A_{\nu}^{3}-2 A^{3 \mu} A^{a \nu} \partial_{\mu} A_{\nu}^{a}\right] .
\end{aligned}
$$

Hence when the vev $v \neq 0$, this set-up spontaneosly breaks the non-abelian gauge symmetry. It not only provides a mass to the three gauge bosons but also ghost-free derivative self-interactions among them that corresponds to a non-abelian generalization of [7]. 
Moreover, it introduces new higher-dimensional couplings (with or without derivatives) between the Higgs field and the vector, proportional to the coupling constant $\beta$. The Lagrangian (3.16) is expressed in unitary gauge: if we were to re-introduce the would-be Goldstone bosons, we would find new interactions between them and the Higgs field, that in an appropriate decoupling limit leads to a theory of multi-Galileons, generalizing the findings of the previous section.

\section{Conclusions}

We presented a Higgs mechanism for spontaneously breaking a gauge symmetry, to obtain the non-linear derivative vector self-interactions recently studied in $[7,8]$, and extended the discussion to a case with non-abelian symmetry. After symmetry breaking, the resulting theory contains the desired vector self-interactions, and in addition new ghost-free derivative interactions between the Higgs and the vector bosons. We studied some of the features of the resulting set-up. We showed that the Lagrangian controlling the would-be Goldstone boson of this theory obtains a Galileon structure in an appropriate decoupling limit. Interestingly, in the same limit the would-be Goldstone boson also acquires derivative couplings with the physical Higgs, that combine in such a way to form a bi-Galileon system with fixed coefficients, determined by gauge invariance. This suggests that, once we introduce an appropriate source, a Vainshtein mechanism should actively screen it from both the longitudinal mode of the vector and the Higgs field of the full theory.

Our results can be further developed and extended, both from a phenomenological and a theoretical perspective. From the point of view of phenomenology, it is known that vector theories with derivative self-interactions can have interesting cosmological applications [7, 18]. It would be interesting to understand whether the new interactions associated with the Higgs scalar can improve the strong-coupling issues of cosmological solutions driving acceleration [18], in a way resembling the quasi-dilaton extension of massive gravity [19]. Namely, the inclusion of an additional degree of freedom together with its special structure suggests that the cosmology of our model could have a far richer phenomenology. Also, it would be interesting to understand whether the Vainshtein-like screening mechanism that suppresses the effect of the longitudinal vector mode [7] is somehow modified by the interactions with the Higgs scalar, possibly offering new suggestions for testing the theory.

The utility of the Vainshtein mechanism opens up the number of ways we can add couplings with other fields. Without a screening mechanism, we would have to confine our theory to a dark sector such that there are no detectable interactions with the Standard Model. At the end of section 2 we briefly discussed how to couple the Higgs field to external matter. It remains to be investigated whether our theory can be coupled to Standard Model fields in a gauge invariant way such that the interactions with the longitudinal mode of the vector and Higgs are screened. This would necessarily entail addressing an open problem in the field. That is, whether suitable Vainshtein mechanisms are possible beyond the very symmetrical and static matter distributions studied so far. Specifically, we would like to consider whether currents formed from Standard Model particles, which usually source the electromagnetic field, could source in a non-linear way, the extra modes in the infrared 
sector of our theory. In a different footing, one needs to understand whether our Higgs selfinteractions can find some new applications in particle physics model building, exploring the possibility that the Higgs field we discussed corresponds to the Standard Model Higgs.

From a more theoretical point of view, our set-up might be regarded as a possible step towards UV-completions of theories closely related to Galileons. Whether our Higgs construction can improve some of the high energy features of the theory and have some role when studying quantum effects remains an open problem. However we find it intriguing that our theory still exhibits a bi-Galileon structure in an appropriate decoupling limit, showing that the Higgs field does not ruin the Galileon symmetry. It is also possible that the structure of the higher dimensional Higgs operators we considered is somehow protected by non-renormalization theorems similar to the ones that apply to Galileon theories. These observations might serve as a guide towards finding Higgs mechanisms for other theories related to Galileons, as for example massive gravity.

\section{Acknowledgments}

It is a pleasure to thank Dario Cannone, Javier Chagoya, Kevin Falls, Andrew Matas and Nicholas Ondo for useful discussions. MH is support by a U.K. Science and Technology Facilities Council (STFC) research studentship. KK is supported by the STFC grants ST/K00090/1 and ST/L005573/1. GT thanks the STFC for financial support through the grant ST/H005498/1.

\section{A Consistency of our Higgs higher-dimensional interactions}

In this appendix we would like to develop some arguments aimed to show that the Higgs interactions contained in Lagrangians (2.13)-(2.15) are consistent, in the sense that they are free of ghost degrees of freedom. We specialize to the case of abelian symmetry breaking, but the same arguments can be straightforwardly extended to the non-abelian case. The interactions in eqs. (2.13)-(2.15) are built in terms of totally antisymmetric $\varepsilon$-tensors. Once expanding the covariant derivatives acting on the Higgs field, and decomposing the Higgs in norm and phase as in the main text, we find that there can arise three kinds of possibly dangerous combinations:

$$
\begin{aligned}
& \epsilon^{\alpha_{1} \alpha_{2} \ldots} \epsilon_{\beta_{1} \beta_{2} \ldots} \partial_{\alpha_{1}} \partial^{\beta_{1}} \varphi \partial_{\alpha_{2}} \varphi \partial^{\beta_{2}} \varphi \ldots \\
& \epsilon^{\alpha_{1} \alpha_{2} \ldots} \epsilon_{\beta_{1} \beta_{2} \ldots} A_{\alpha_{1}} A^{\beta_{1}} \partial_{\alpha_{2}} \varphi \partial^{\beta_{2}} \varphi \ldots \\
& \epsilon^{\alpha_{1} \alpha_{2} \ldots} \epsilon_{\beta_{1} \beta_{2} \ldots} \partial^{\beta_{1}} A_{\alpha_{1}} \varphi \partial_{\alpha_{2}} \varphi \partial^{\beta_{2}} \varphi \ldots
\end{aligned}
$$

where the dots contain additional pieces, of the same type as the above, or other contributions that contain single or no derivatives of $\varphi$ - always contracted with the $\varepsilon$-tensor. Interactions as the ones listed in (A.1)-(A.3), when appearing in the Lagrangian, are $a$ priori dangerous because they contain second derivatives acting on the scalar $\varphi$, and/or the gauge potential $A_{\mu}$. We have to ensure that the corresponding equations of motion do not contain more than two space-time derivatives of the fields involved. Moreover, the 
equation of motion for $A_{0}$ should not contain time derivatives acting on $A_{0}$ itself, so to ensure that $A_{0}$ is a constraint. These requirements, together with the positivity of the kinetic terms, are sufficient to ensure the absence of ghosts.

Interactions as (A.1) are the familiar scalar Galileon interactions [5]: the structure of the $\varepsilon$-tensors does not allow them to generate higher space-time derivatives in their equations of motion. Indeed, the equations of motion for a scalar field $\varphi$ can certainly lead to derivatives acting on the first part, $\partial_{\alpha_{1}} \partial^{\beta_{1}} \varphi$, of (A.1) - as for example contributions like

$$
\epsilon^{\alpha_{1} \alpha_{2} \ldots} \epsilon_{\beta_{1} \beta_{2} \ldots} \partial_{\alpha_{2}} \partial^{\beta_{2}} \partial_{\alpha_{1}} \partial^{\beta_{1}} \varphi \ldots \quad \text { or } \quad \epsilon^{\alpha_{1} \alpha_{2} \ldots} \epsilon_{\beta_{1} \beta_{2} \ldots} \partial_{\alpha_{2}} \partial_{\alpha_{1}} \partial^{\beta_{1}} \varphi \ldots
$$

But the $\varepsilon$-tensor makes them vanishing: the operator $\partial_{\alpha_{1}} \partial_{\alpha_{2}}$ is symmetric on its indexes, and gives zero when contracted with the $\epsilon^{\alpha_{1} \alpha_{2} \ldots}$. This fact is familiar and was developed in [20]. Similar arguments can be made to show that (A.2), (A.3) cannot contribute to the equation of motion for $A_{0}$ with terms containing the time derivative of $A_{0}$ itself (see also $[7,8])$. Since $A_{\mu}$ is always contracted with the $\varepsilon$-tensor, it is simple to convince oneself that the only possibly dangerous contributions from the equation of motion of $A_{0}$ - that is the ones that might have time derivatives acting on $A_{0}$ - are pieces that contain first or second derivatives acting on the gauge potential, as

$$
\epsilon^{0 \cdots} \epsilon_{\beta_{1} \beta_{2} \ldots} \partial^{\beta_{1}} A^{\beta_{2}}, \quad \text { or } \quad \epsilon^{0 \alpha_{2} \cdots} \epsilon_{\beta_{1} \ldots} \partial^{\beta_{1}} A_{\alpha_{2}}, \quad \text { or } \quad \epsilon^{0 \alpha_{2} \cdots} \epsilon_{\beta_{1} \beta_{2} \ldots} \partial_{\alpha_{2}} \partial^{\beta_{1}} A^{\beta_{2}} \text {. }
$$

In the first option, the index $\beta_{1}$ and $\beta_{2}$ can not simultaneously take the value zero, due to the antisymmetric property of the $\varepsilon$-tensor, hence this contribution vanishes for the possibly dangerous case. A similar argument exists for the second and third option. The crucial fact is that one of the indexes of the $\varepsilon$-tensor is already fixed to be zero since we are evaluating the equation of motion for $A_{0}$; hence, $\alpha_{2} \neq 0$ and we cannot have time derivatives acting on $A_{0}$.

\section{B Ghost free scalar-vector interactions}

\section{B.1 Bi-Galileons}

We wish to find ghost free derivative couplings between a scalar $\pi$ and a vector field $A_{\mu}$. In order to achieve this, we will find it useful to first consider 'bi-Galileon' interactions. BiGalileons are an extension to two scalar fields of the original Galileon theory first introduced to cosmology in [5]. (Their properties, however, were first discussed in [21] for a rather different purpose.)

A Galileon is a scalar field $\pi$ the action of which is invariant under Galilean shifts in its field space, $\pi \rightarrow \pi+b_{\mu} x^{\mu}+c$. They have the property that although their actions contain both first and second order derivatives, their equations of motion are of second order only. Furthermore, it was shown in $[5,21]$ that, up to total derivatives, there is a unique term for each order in the field $\pi$ up to $n+1$, where $n$ is the dimension of the space-time. This is due to the fact that each nontrivial derivative term is associated with one Cayley invariant of the matrix $\partial_{\mu} \partial_{\nu} \pi$. 
We make use of the Levi-Civita epsilon tensor to write the Lagrangian for the Galileons in a compact form [20]. Using the following property:

$$
\varepsilon_{\gamma_{1} \ldots \gamma_{D-n} \alpha_{1} \ldots \alpha_{n}} \varepsilon^{\gamma_{1} \ldots \gamma_{D-n} \beta_{1} \ldots \beta_{n}}=-(D-n) ! n ! \delta_{\alpha_{1} \ldots \alpha_{n}}^{\left[\beta_{1} \ldots \beta_{n}\right]}
$$

where the square brackets represent normalised anti-symmetric permutations, we can write the Galileon Lagrangians as:

$$
\begin{aligned}
& \mathcal{L}_{1}=\pi \\
& \mathcal{L}_{2}=\frac{1}{3 !} \varepsilon^{\mu_{1} \nu \lambda \gamma} \varepsilon_{\nu \lambda \gamma}^{\mu_{2}} \pi_{\mu_{1}} \pi_{\mu_{2}}:=\mathcal{E}_{(2)} \pi_{1} \pi_{2} \\
& \mathcal{L}_{3}=\frac{1}{2 !} \varepsilon^{\mu_{1} \mu_{3} \nu \lambda} \varepsilon^{\mu_{2} \mu_{4}}{ }_{\nu \lambda} \pi_{\mu_{1}} \pi_{\mu_{2}}\left(\pi_{\mu_{3} \mu_{4}}\right):=\mathcal{E}_{(4)} \pi_{1} \pi_{2}\left(\pi_{34}\right) \\
& \mathcal{L}_{4}=\varepsilon^{\mu_{1} \mu_{3} \mu_{5} \nu} \varepsilon^{\mu_{2} \mu_{4} \mu_{6}}{ }_{\nu} \pi_{\mu_{1}} \pi_{\mu_{2}}\left(\pi_{\mu_{3} \mu_{4}} \pi_{\mu_{5} \mu_{6}}\right):=\mathcal{E}_{(6)} \pi_{1} \pi_{2}\left(\pi_{34} \pi_{56}\right) \\
& \mathcal{L}_{5}=\varepsilon^{\mu_{1} \mu_{3} \mu_{5} \mu_{7}} \varepsilon^{\mu_{2} \mu_{4} \mu_{6} \mu_{8}} \pi_{\mu_{1}} \pi_{\mu_{2}}\left(\pi_{\mu_{3} \mu_{4}} \pi_{\mu_{5} \mu_{6}} \pi_{\mu_{7} \mu_{8}}\right):=\mathcal{E}_{(8)} \pi_{1} \pi_{2}\left(\pi_{34} \pi_{56} \pi_{78}\right)
\end{aligned}
$$

Where we have defined $\mathcal{E}_{2 n}^{1234 \ldots}=\frac{1}{(D-n) !} \varepsilon^{135 \ldots \nu_{1} \nu_{2} \ldots \nu_{D-n}} \varepsilon^{246 \ldots}{ }_{\nu_{1} \nu_{2} \ldots \nu_{D-n}}$ which has been written in short hand as $\mathcal{E}_{(2 n)}$ and the numbers are short hand for labeled indices: $\left\{\mu_{1} \mu_{2} \ldots\right\}$. Furthermore, we have that $\pi_{\mu_{1} \ldots \mu_{n}} \equiv \partial_{\mu_{n}} \ldots \partial_{\mu_{1}} \pi$.

With this notation it is very easy to see that the variation of these Lagrangians would never have higher than two derivatives. For instance, taking the variation of $\mathcal{L}_{5}$ gives us:

$$
\begin{aligned}
0 & =\delta \mathcal{S}_{5}=\int \mathrm{d}^{4} x \delta \mathcal{L}_{5} \\
& =\int \mathrm{d}^{4} x \mathcal{E}_{(8)}\left[2 \delta \pi_{1} \pi_{2}\left(\pi_{34} \pi_{56} \pi_{78}\right)+3 \pi_{1} \pi_{2}\left(\delta \pi_{34} \pi_{56} \pi_{78}\right)\right] \\
& =\int \mathrm{d}^{4} x \mathcal{E}_{(8)}\left[-2 \partial_{1}\left(\pi_{2} \pi_{34} \pi_{56} \pi_{78}\right)-3 \partial_{3} \partial_{4}\left(\pi \pi_{12} \pi_{56} \pi_{78}\right)\right] \delta \pi \\
& =-5 \int \mathrm{d}^{4} x \mathcal{E}_{(8)}\left(\pi_{12} \pi_{34} \pi_{56} \pi_{78}\right)
\end{aligned}
$$

Where we have integrated by parts and found that the only term to survive the summation with the totally antisymmetric tensor $\mathcal{E}_{(8)}$ has, indeed, only derivatives of second order.

Bi-Galileons were first introduced in a general setting in [12] and were treated in depth in [13]. The action for the two scalar fields $\pi$ and $h$, is invariant under separate Galilean transformations: $\pi \rightarrow \pi+b_{\mu}^{(\pi)} x^{\mu}+c^{(\pi)}$ and $h \rightarrow h+b_{\mu}^{(h)} x^{\mu}+c^{(h)}$. Furthermore, the equations of motion for both fields are exactly second order in their derivatives. We use the notation introduced above and follow the methods outlined in [12].

First we enforce a symmetry relation. That is, $\mathcal{L}_{h \pi}=\mathcal{L}_{\pi h}$ with $h \leftrightarrow \pi$. I.e.

$$
\varepsilon^{\mu \nu \rho \lambda} \varepsilon_{\alpha \beta \gamma \lambda} \pi_{\mu} h^{\alpha}\left(h_{\nu}^{\beta} h_{\rho}^{\gamma}\right) \rightarrow \varepsilon^{\mu \nu \rho \lambda} \varepsilon_{\alpha \beta \gamma \lambda} h_{\mu} \pi^{\alpha}\left(\pi_{\nu}^{\beta} \pi_{\rho}^{\gamma}\right)
$$

It will be important to remember this choice when we substitute the vector for one of the Galileons. 
The general Lagrangian can be written as the sum of the following sub-Lagrangians: $\mathcal{E}_{(8)}$ :

$$
\begin{aligned}
& \alpha_{(5,0)} \mathcal{L}_{(5,0)}=\alpha_{(5,0)} \mathcal{E}_{(8)} h_{1} h_{2}\left(h_{34} h_{56} h_{78}\right) \\
& \alpha_{(4,1)} \mathcal{L}_{(4,1)}=\alpha_{(4,1)} \mathcal{E}_{(8)} h_{1} \pi_{2}\left(h_{34} h_{56} h_{78}\right) \\
& \alpha_{(3,2)} \mathcal{L}_{(3,2)}=\alpha_{(3,2)} \mathcal{E}_{(8)} h_{1} \pi_{2}\left(\pi_{34} h_{56} h_{78}\right)
\end{aligned}
$$

$\mathcal{E}_{(6)}:$

$$
\begin{aligned}
& \alpha_{(4,0)} \mathcal{L}_{(4,0)}=\alpha_{(4,0)} \mathcal{E}_{(6)} h_{1} h_{2}\left(h_{34} h_{56}\right) \\
& \alpha_{(3,1)} \mathcal{L}_{(3,1)}=\alpha_{(3,1)} \mathcal{E}_{(6)} h_{1} \pi_{2}\left(h_{34} h_{56}\right) \\
& \alpha_{(2,2)} \mathcal{L}_{(2,2)}=\alpha_{(2,2)} \mathcal{E}_{(6)} h_{1} \pi_{2}\left(\pi_{34} h_{56}\right)
\end{aligned}
$$

$\mathcal{E}_{(4)}:$

$$
\begin{aligned}
& \alpha_{(3,0)} \mathcal{L}_{(3,0)}=\alpha_{(3,0)} \mathcal{E}_{(4)} h_{1} h_{2}\left(h_{34}\right) \\
& \alpha_{(2,1)} \mathcal{L}_{(2,1)}=\alpha_{(2,1)} \mathcal{E}_{(4)} h_{1} \pi_{2}\left(h_{34}\right)
\end{aligned}
$$

$\mathcal{E}_{(2)}$ :

$$
\begin{aligned}
& \alpha_{(2,0)} \mathcal{L}_{(2,0)}=\alpha_{(2,0)} \mathcal{E}_{(2)} h_{1} h_{2} \\
& \alpha_{(1,1)} \mathcal{L}_{(1,1)}=\alpha_{(1,1)} \mathcal{E}_{(2)} h_{1} \pi_{2}
\end{aligned}
$$

$\mathcal{E}_{(0)}:$

$$
\alpha_{(1,0)} \mathcal{L}_{(1,0)}=\alpha_{(1,0)} \mathcal{E}_{(0)} h
$$

Where for each sub-Lagrangian we have the corresponding symmetrical exchange of the two fields: $\beta_{(m, n)} \mathcal{L}_{(m, n)}=\beta_{(m, n)} \mathcal{E}_{(2(m+n-1))} \pi_{1} h_{2}\left(\pi_{34} \ldots\right)$.

\section{B.2 Bi-vectors and the scalar-vector Lagrangian}

The above bi-Galileon terms can be identified as the decoupling limit of an interaction between a scalar and a vector. Due to their special properties, these interactions cannot induce a ghostly fourth mode, (ghost free scalar-vector interactions were discussed in a different context in [22]). We construct these interaction terms by first considering the products of two vectors, $X_{\mu}=\left\{A_{\mu}, B_{\mu}\right\}$ with their derivatives, $X_{\mu \nu} \equiv \partial_{\mu} X_{\nu}=\left\{\partial_{\mu} A_{\nu}, \partial_{\mu} B_{\nu}\right\}$ and then substituting $B_{\mu} \equiv \partial_{\mu} h$ :

$$
\mathcal{L}_{\text {bi-vector }}=\mathcal{E}_{(2 n)} X_{1} X_{2}\left(X_{\{34\}} \ldots X_{\{2 n-12 n\}}\right)
$$

Where we use \{\}$:=($ ) or [] to indicate symmetric and anti-symmetric combinations respectfully.

When we constructed the Galileons above we relied on the fact that the indices associated with the partial derivatives acting on the scalar field commute (i.e. $\pi_{\mu \nu}=\pi_{\nu \mu}$ ). 
For vectors, however, this is not true as the indices associated with the vector cannot be commuted (anti-commuted) with the indices associated with the partial derivative (i.e. $\left.\partial_{\mu} A_{\nu} \neq \partial_{\nu} A_{\mu}\right)$ and thus we need to take into account the new combinations that are possible. This subtlety was discussed for a single gauge field in [8] where it was found that one extra parameter is needed for both the quartic and quintic vector Galileons. Furthermore, notice that, in the decoupling limit, these separate scalar-vector interactions converge to the same bi-Galileon term as they are related by the symmetry outlined above. Although we add some redundancy due to some terms differing only by a total derivative, it is convenient to construct our Lagrangian by choosing $X_{n}:=a A_{n}+b B_{n}$ and $X_{\{n m\}}:=a A_{\{n m\}}+b B_{\{n m\}}$.

In order to make contact with the main text, in the following we consider only terms up to cubic order in the fields. In such a case, we find that the terms with $X_{[n m]}$ cancel and we have:

$$
\begin{aligned}
\mathcal{L}_{\text {bi-vector }}^{(3)}= & \mathcal{E}_{(4)} X_{1} X_{2}\left(X_{\{(34)\}}\right) \\
= & \mathcal{E}_{(4)}\left(a A_{1}+b B_{1}\right)\left(a A_{2}+b B_{2}\right)\left(a A_{(34)}+b B_{(34)}\right) \\
= & \mathcal{E}_{(4)}\left\{a^{3} A_{1} A_{2}\left(A_{(34)}\right)+a^{2} b\left[A_{1} A_{2}\left(B_{(34)}\right)+2 A_{1} B_{2}\left(A_{(34)}\right)\right]\right. \\
& \left.\quad \quad \quad \text { exchange }\left\{a A_{n}, a A_{(n m)}\right\} \longleftrightarrow\left\{b B_{n}, b B_{(n m)}\right\}\right\}
\end{aligned}
$$

Substituting $\partial_{\mu} h$ for $B_{\mu}$ gives us the cubic scalar-vector interactions:

$$
\begin{aligned}
\alpha_{(3,0)} \mathcal{L}_{(3,0)}^{s v} & =\alpha_{(3,0)} \mathcal{E}_{(4)} A_{1} A_{2}\left(A_{(34)}\right) \\
\alpha_{(2,1)} \mathcal{L}_{(2,1)}^{s v} & =\alpha_{(2,1)} \mathcal{E}_{(4)} A_{1} A_{2}\left(h_{34}\right) \\
\alpha_{(2,1)^{\prime}} \mathcal{L}_{(2,1)^{\prime}}^{s v} & =\alpha_{(2,1)^{\prime}} \mathcal{E}_{(4)} A_{1} h_{2}\left(A_{(34)}\right) \\
\beta_{(0,3)} \mathcal{L}_{(0,3)}^{s v} & =\beta_{(0,3)} \mathcal{E}_{(4)} h_{1} h_{2}\left(h_{34}\right) \\
\beta_{(1,2)} \mathcal{L}_{(1,2)}^{s v} & =\beta_{(1,2)} \mathcal{E}_{(4)} h_{1} h_{2}\left(A_{(34)}\right) \\
\beta_{(1,2)^{\prime}} \mathcal{L}_{(1,2)^{\prime}}^{s v} & =\beta_{(1,2)^{\prime}} \mathcal{E}_{(4)} h_{1} A_{2}\left(h_{34}\right)
\end{aligned}
$$

Where,

$$
\begin{cases}\alpha_{(n, m)}=a^{n} b^{m} \text { and } \alpha_{(n, m)^{\prime}}=2 a^{n} b^{m} & \text { if } n>m, \\ \beta_{(n, m)}=a^{n} b^{m} \text { and } \beta_{(n, m)^{\prime}}=2 a^{n} b^{m} & \text { if } n<m .\end{cases}
$$

These interactions return to the above cubic bi-Galileon terms in the appropriate decoupling limit, or rather, under substituting $A_{\mu}$ with $\partial_{\mu} \pi$. Lastly, for the convenience of the reader, we expand below the terms which correspond to the interactions generated in the main text:

$$
\begin{aligned}
\alpha_{(3,0)} \mathcal{L}_{(3,0)}^{s v} & =\alpha_{(3,0)} \mathcal{E}_{(4)} A_{1} A_{2}\left(A_{34}\right)=-\alpha_{(3,0)} 2 ! \delta_{\mu_{1} \mu_{3}}^{\left[\mu_{2} \mu_{4}\right]} A^{\mu_{1}} A_{\mu_{2}}\left(A_{\mu_{4}}^{\mu_{3}}\right) \\
& =-\alpha_{(3,0)} 2 A^{2}(\partial \cdot A)+\alpha_{(3,0)} \frac{1}{2} A_{\mu}\left(\partial^{\mu} A^{\nu}+\partial^{\nu} A^{\mu}\right) A_{\nu} \\
& =-\frac{5}{2} \alpha_{(3,0)} A^{2}(\partial \cdot A)
\end{aligned}
$$




$$
\begin{aligned}
\beta_{(1,2)} \mathcal{L}_{(1,2)}^{s v} & =\beta_{(1,2)} \mathcal{E}_{(4)} h_{1} A_{2}\left(h_{34}\right)=-\beta_{(1,2)} 2 ! \delta_{\mu_{1} \mu_{3}}^{\left[\mu_{2} \mu_{4}\right]} h^{\mu_{1}} A_{\mu_{2}}\left(h_{\mu_{4}}^{\mu_{3}}\right) \\
& =-\beta_{(1,2)}\left(\partial^{\mu} h A_{\mu}(\square h)-\partial^{\mu} h A^{\nu}\left(\partial_{\mu} \partial_{\nu} h\right)\right) \\
& =-\beta_{(1,2)}\left(\partial_{\mu} h A^{\mu}(\square h)+\partial^{\nu} \partial_{\mu} h A_{\nu} \partial^{\mu} h+\partial_{\mu} h \partial^{\nu} A_{\nu} \partial^{\mu} h\right) \\
& =-\beta_{(1,2)}\left((\partial h)^{2} \partial \cdot A-\partial_{\nu} h \partial^{\nu} A^{\mu} \partial_{\mu} h\right)
\end{aligned}
$$

Open Access. This article is distributed under the terms of the Creative Commons Attribution License (CC-BY 4.0), which permits any use, distribution and reproduction in any medium, provided the original author(s) and source are credited.

\section{References}

[1] E.J. Copeland, M. Sami and S. Tsujikawa, Dynamics of dark energy, Int. J. Mod. Phys. D 15 (2006) 1753 [hep-th/0603057] [INSPIRE].

[2] T. Clifton, P.G. Ferreira, A. Padilla and C. Skordis, Modified gravity and cosmology, Phys. Rept. 513 (2012) 1 [arXiv:1106.2476] [InSPIRE].

[3] J. Khoury and A. Weltman, Chameleon fields: awaiting surprises for tests of gravity in space, Phys. Rev. Lett. 93 (2004) 171104 [astro-ph/0309300] [INSPIRE].

[4] A.I. Vainshtein, To the problem of nonvanishing gravitation mass, Phys. Lett. B 39 (1972) 393 [INSPIRE].

[5] A. Nicolis, R. Rattazzi and E. Trincherini, The Galileon as a local modification of gravity, Phys. Rev. D 79 (2009) 064036 [arXiv:0811.2197] [InSPIRE].

[6] C. de Rham, G. Gabadadze and A.J. Tolley, Resummation of massive gravity, Phys. Rev. Lett. 106 (2011) 231101 [arXiv:1011.1232] [INSPIRE].

[7] G. Tasinato, Cosmic acceleration from Abelian symmetry breaking, JHEP 04 (2014) 067 [arXiv:1402.6450] [INSPIRE].

[8] L. Heisenberg, Generalization of the Proca action, JCAP 05 (2014) 015 [arXiv:1402.7026] [INSPIRE].

[9] K. Kamada, T. Kobayashi, M. Yamaguchi and J. Yokoyama, Higgs G-inflation, Phys. Rev. D 83 (2011) 083515 [arXiv:1012.4238] [INSPIRE].

[10] S.-Y. Zhou and E.J. Copeland, Galileons with gauge symmetries, Phys. Rev. D 85 (2012) 065002 [arXiv:1112.0968] [INSPIRE].

[11] M.E. Peskin and D.V. Schroeder, An introduction to quantum field theory, Addison-Wesley, Reading U.S.A. (1995).

[12] C. Deffayet, S. Deser and G. Esposito-Farese, Arbitrary p-form Galileons, Phys. Rev. D 82 (2010) 061501 [arXiv:1007.5278] [InSPIRE].

[13] A. Padilla, P.M. Saffin and S.-Y. Zhou, Bi-Galileon theory I: motivation and formulation, JHEP 12 (2010) 031 [arXiv:1007.5424] [INSPIRE].

[14] M.A. Luty, M. Porrati and R. Rattazzi, Strong interactions and stability in the DGP model, JHEP 09 (2003) 029 [hep-th/0303116] [INSPIRE].

[15] A. Nicolis and R. Rattazzi, Classical and quantum consistency of the DGP model, JHEP 06 (2004) 059 [hep-th/0404159] [INSPIRE]. 
[16] J.D. Bekenstein, The relation between physical and gravitational geometry, Phys. Rev. D 48 (1993) 3641 [gr-qc/9211017] [InSPIRE].

[17] M. Maggiore, A modern introduction to quantum field theory, Oxford Series in Physics 12, Oxford University Press, Oxford U.K. (2005) [ISBN:0 19 852073 5].

[18] G. Tasinato, A small cosmological constant from Abelian symmetry breaking, Class. Quant. Grav. 31 (2014) 225004 [arXiv: 1404.4883] [INSPIRE].

[19] G. D'Amico, G. Gabadadze, L. Hui and D. Pirtskhalava, Quasidilaton: theory and cosmology, Phys. Rev. D 87 (2013) 064037 [arXiv:1206.4253] [InSPIRE].

[20] C. Deffayet, S. Deser and G. Esposito-Farese, Generalized Galileons: all scalar models whose curved background extensions maintain second-order field equations and stress-tensors, Phys. Rev. D 80 (2009) 064015 [arXiv:0906. 1967] [inSPIRE].

[21] D.B. Fairlie, J. Govaerts and A. Morozov, Universal field equations with covariant solutions, Nucl. Phys. B 373 (1992) 214 [hep-th/9110022] [INSPIRE].

[22] N. Khosravi, Spontaneous scalar-vector Galileons from a Weyl biconnection model, Phys. Rev. D 89 (2014) 124027 [arXiv: 1404.7503] [INSPIRE]. 